\title{
INFLUÊNCIA DE DIFERENTES SISTEMAS DE USO DO SOLO NA DENSIDADE E DIVERSIDADE DE MACROFAUNA EM BOA VISTA-RR
}

\section{INFLUENCE OF DIFFERENT SOIL USE SYSTEMS ON THE DENSITY AND DIVERSITY OF MACROFAUNA IN BOA VISTA-RR}

\author{
Tatiane Patrícia Silvério Ribeiro ${ }^{1}$ \\ Ariane Silva Magalhães \\ Cleudimar Brito dos Santos \\ Ana Cristina Alencar \\ Plínio Henrique Oliveira Gomide ${ }^{2}$ \\ Járisson Cavalcante Nunes ${ }^{3}$
}

\begin{abstract}
RESUMO: A macrofauna edáfica pode ser utilizada como indicadora da qualidade do solo. Nesse sentido, um levantamento foi realizado no município de Boa Vista, Roraima, com o objetivo de avaliar a influência de diferentes sistemas de uso do solo na macrofauna edáfica. Foram selecionadas quatro áreas: área de savana natural, área com produção de frutíferas consorciada com uso de práticas agroecológicas, área de produção de hortaliças convencional e uma área degradada. As amostras de solo foram coletadas no mês de maio de 2018 , em monólitos de $25 \times 25 \mathrm{~cm}$ e profundidade de 20 $\mathrm{cm}$ sendo 05 amostras em cada ambiente, totalizando 20 parcelas experimentais, seguindo o método Tropical Soil Biology and Fertility. A macrofauna edáfica nos diferentes sistemas de uso do solo foi identificada por grupos taxonômicos. Os grupos mais abundantes nas áreas amostradas foram Isoptera, Isopoda, Chilopoda, Oligochaeta, Coleoptera e Hymenoptera, este último com o maior número encontrado. Conclui-se que a macrofauna mostrou-se sensível ao manejo empregado nas áreas servindo como bioindicador da qualidade do solo nestes ambientes. A área de frutíferas consorciada com uso de práticas agroecológicas apresentou maior diversidade, equitabilidade e riqueza, em relação às demais áreas estudadas.
\end{abstract}

Palavras-chave: Macrofauna edáfica, bioindicador, qualidade do solo.

ABSTRACT: The edaphic macrofauna can be used as an indicator of soil quality. In this sense, a survey was carried out in the municipality of Boa Vista, Roraima, with the objective of evaluating the influence of different soil use systems on the edaphic macrofauna. Four areas were selected: natural savanna area, area with fruit production consorted with agroecological practices, conventional vegetable production area and degraded area. Soil samples were collected in May of 2018, in monoliths of $25 \times 25 \mathrm{~cm}$ and depth of $20 \mathrm{~cm}$, with 05 samples in each environment, totaling 20 experimental plots, following the Tropical Soil Biology and Fertility method. The edaphic

\footnotetext{
${ }^{1}$ Mestrandas em Agroecologia, Programa de Pós-Graduação em Agroecologia, Universidade Estadual de Roraima - UERR. E-mail: arianemagalhaes.boavista@gmail.com, tpatriciasr@gmail.com, britosgv@hotmail.com, anacristinalovealencar@gmail.com

${ }^{2}$ Professor do Programa de Pós-Graduação em Agroecologia da UERR. E-mail: pliniogomide@gmail.com

${ }^{3}$ Programa Nacional de Pós-Doutorado/Capes, Programa de Pós-Graduação em Agroecologia, Universidade Estadual de Roraima- UERR. E-mail: jarissonagro@ hotmail.com
} 
macrofauna in the different land use systems was identified by taxonomic groups. The most abundant groups in the sampled areas were Isoptera, Isopoda, Chilopoda, Oligochaeta, Coleoptera and Hymenoptera, the latter with the highest number found. It was concluded that the macrofauna was sensitive to the management used in the areas serving as a bioindicator of soil quality in these environments. The area of fruit trees intercropped with the use of agroecological practices presented greater diversity, equitability and richness in relation to the other areas studied.

Key words: Soil macrofauna, bioindicator, soil quality.

\section{INTRODUÇÃO}

A fruticultura e a olericultura tem se expandido ao longo dos anos e sua produção tem grande importância na geração de renda e emprego, sendo considerada uma atividade típica da agricultura familiar de Roraima (DUARTE et al., 2009). A produção de olerícolas está estabelecida na área urbana e periurbana da cidade de Boa Vista, onde são cultivadas diversas espécies de valor econômico garantindo geração de emprego e renda para a agricultura familiar em pequena escala (QUERINO et al., 2008).

A prática de manejo utilizado pela maioria dos produtores de hortaliças e frutíferas de Boa Vista- RR é proveniente da agriculta convencional, embora já tenha um número considerável de produtores em transição para a agricultura orgânica ou praticando o manejo agroecológico (SILVA, 2016).

O manejo adotado no solo pode conservá-lo, melhorar suas propriedades físicas, químicas e biológicas, mas também pode contribuir no processo de erosão, perda de matéria orgânica e de nutrientes. No solo ocorrem muitos processos biológicos que são importantes. Pode-se destacar, entre esses processos, a ciclagem de nutrientes, o controle biológico de pragas e de doenças, a decomposição de resíduos tóxicos, a purificação da água e a produção de alimentos, mediados pelos organismos do solo (KORASAKI et al., 2013).

Existe uma grande variedade de espécies no solo com tamanhos e metabolismos diversos e são responsáveis por inúmeras funções no ambiente edáfico. Dentre os invertebrados do solo, a macrofauna é fundamental ao funcionamento do ecossistema, pois, além de afetar a produção primária de maneira direta e indireta, ocupa todos os níveis tróficos na cadeia alimentar do solo (SILVA et al., 2007; ALMEIDA et al., 2015, SOUZA et al, 2017). A macrofauna compreende organismos com tamanho entre 2 a 10 
mm, e são capazes de remover o solo, abrindo galerias que permitem fazer ligações entre os horizontes do solo, como são o caso das minhocas, formigas e cupins, que são considerados os principais modificadores do sistema solo (BRADY; WEILL, 2013).

Atualmente procuram-se práticas de manejo menos agressivas ao meio ambiente e estudos com foco na estrutura da comunidade de invertebrados no solo que visam identificar as opções de manejo que possam aperfeiçoar suas atividades para o funcionamento do ecossistema e manutenção da boa qualidade do solo nas práticas agrícolas são necessários.

Informações sobre a biota do solo em áreas implantadas com diferentes culturas podem subsidiar o planejamento para a melhoria das características edáficas, pois além de avaliar as transformações exercidas pela intervenção antrópica, o estudo da macrofauna edáfica serve como monitoramento das modificações nestes ambientes fornecendo também, informações sobre a conservação e manutenção do equilíbrio dos agroecossistemas (SOUSA et al., 2016). Estudos dessa natureza em Roraima são escassos ou inexistentes. Desse modo, o objetivo desse estudo foi avaliar a influência de diferentes sistemas de uso do solo sobre a macrofauna edáfica no município de Boa Vista, Roraima.

\section{MATERIAL E MÉTODOS}

A pesquisa foi realizada durante o mês de maio de 2018, no munícipio de Boa Vista/RR. A classificação Köppen para o clima da região é do tipo Aw (ALVARES et al. 2014), com duas estações climáticas bem definidas, uma chuvosa (abril-setembro) e outra seca (outubro-março) (ARAÚJO et al., 2001). A coleta da macrofauna edáfica foi realizada em maio/2018, no início da estação chuvosa em Boa Vista.

Para realização da pesquisa foram selecionadas quatro áreas circunvizinhas com manejos distintos: 1) savana natural - SN (N 02 ${ }^{\circ} 51,215^{` e} 60^{\circ} 39,122^{`} \mathrm{~W}$ ): corresponde a uma área natural com presença de gramíneas e arbustos como o caimbé (Curatela americana), o mirixi (Byrsonima crassifolia) e o mirixi-cajú (B. coccolobifolia); 2) área de frutíferas consorciada com uso de práticas agroecológicas - AF ( $\mathrm{N} \mathrm{02}$ 47,191 e $60^{\circ}$ 45,81 'W): corresponde a uma área que é cultivada desde 2002 e que a partir de 2009 iniciou-se o plantio de frutíferas. Nessa área, o manejo é realizado com a utilização de práticas agroecológicas, como utilização de resíduos vegetais na produção da compostagem para adubação das plantas de interesse comercial, cobertura vegetal no controle da erosão, consórcio de culturas com açaí (Euterpe oleracea), banana (Musa 
spp.), laranja (Citrus sinensis), além da cobertura morta. 3) área de produção de hortaliças convencional - $\mathrm{HC}\left({\mathrm{N} 02^{\circ}}^{\circ}\right.$ 1,207` e $\left.60^{\circ} 39,137 \mathrm{~W}\right)$ : corresponde a um lote de terras urbano que foi aberto e está em uso desde o ano de 2010 com práticas convencionais para o plantio de hortaliças como cebolinha (Allium fistolosum), alface (Lactuca sativa), coentro (Coriandrum sativum) e couve (Brassica oleracea), uso de fertilizantes e pesticidas sintéticos. 4) área degradada - $\mathrm{AD}\left({\mathrm{N} 02^{\circ}}^{\circ} 47,091^{`}\right.$ e $60^{\circ} 934^{`}$ W): em 2015 foi implantado um canteiro de obras para o asfaltamento das ruas do bairro atualmente abandonado e degradado. Área bem compactada e sem presença de vegetação.

Para a avaliação da macrofauna edáfica, seguiu-se a metodologia recomendada pelo "Tropical Soil Biology And Fertility” (TSBF) (ANDERSON; INGRAM, 1993). Nas quatro áreas estudadas foram coletados cinco monólitos de solo de $25 \times 25 \mathrm{~cm}$ de comprimento e largura e $20 \mathrm{~cm}$ de profundidade, ao acaso, a uma distância de $20 \mathrm{~m}$ entre si. Antes da retirada do monólito de solo, a serapilheira foi amostrada na mesma área. Os monólitos foram destorroados no interior de bandejas plásticas e com o auxílio de pinças, procedeu-se a catação manual dos organismos, que foram colocados em bandejas plásticas. Após a triagem, identificaram-se os macroinvertebrados, ao nível de grandes grupos taxonômicos (STORER et al., 2000), sendo os mesmos separados e contados para determinação da densidade.

O delineamento experimental utilizado foi inteiramente casualizado (DIC), cujos tratamentos corresponderam às quatro áreas amostradas, com cinco repetições em cada área. $\mathrm{Na}$ avaliação do comportamento ecológico da macrofauna, foi mensurado o número total de indivíduos (abundância) e feitas comparações das comunidades nas áreas estudadas utilizando o índice de diversidade de Shannon e o índice de equitabilidade de Pielou (U).

O índice de diversidade de Shannon $(\mathrm{H})$ é definido por:

$$
\begin{aligned}
& \mathrm{H}=- \text { pi.log } \mathrm{PI}(1) \\
& \mathrm{pi}=\mathrm{ni} / \mathrm{N}(2)
\end{aligned}
$$

Em que ni é a densidade de cada grupo; $\mathrm{N}$ é a somatória da densidade de todos os grupos. 
Esse índice assume valores que podem variar de 0 a 5, sendo que o seu declínio é o resultado de uma maior dominância de grupos em detrimento de outros (BEGON et al., 1996).

O Índice de Uniformidade de Pielou (e) é um índice de eqüitabilidade, sendo definido por:

$$
\mathrm{e}=\mathrm{H} / \log \mathrm{S}(3)
$$

Em que H é o índice de Shannon; S é o número de espécies ou grupos.

Os dados de macrofauna obtidos, dada a sua heterogeneidade, foram transformados em $\ln (\mathrm{x}+1)$. Os dados foram submetidos à análise de variância (teste $\mathrm{F})$ e as médias comparadas pelo teste de Tukey a $5 \%$ de probabilidade, utilizando o programa Sisvar (FERREIRA, 2014). Os dados foram transformados em log (x), para reduzir a variação e proporcionar uma variação mais próxima do normal.

\section{RESULTADOS E DISCUSSÃO}

O levantamento da macrofauna edáfica, nas quatro áreas analisadas totalizaram 424 indivíduos, sendo representados por 6 (seis) grupos taxonômicos (Tabela1). Aqueles encontrados em maior quantidade foram da família Hymenoptera (formigas) e da ordem Oligochaeta (minhocas), seguido da classe Coleoptera (besouros), sendo a Hymenoptera a mais frequente (257indivíduos) nas áreas analisadas.

Tabela 1- Distribuição de número de indivíduos de acordo com o grupo taxonômico em área de savana natural (SN), área de frutíferas consorciada com uso de práticas agroecológicas (AF), área de produção de hortaliças convencional (HC) e área degradada (AD) no município de Boa Vista-RR, em maio de 2018.

\begin{tabular}{|c|c|c|c|c|c|}
\hline $\begin{array}{c}\text { Grupo } \\
\text { Taxonômico }\end{array}$ & $\mathrm{SN}$ & $\mathrm{AF}$ & $\mathrm{HC}$ & $\mathrm{AD}$ & Total \\
\hline & \multicolumn{5}{|c|}{ Número de indivíduos } \\
\hline Coleoptera & 19 & 22 & - & - & 41 \\
\hline Isopoda & 4 & 4 & - & - & 8 \\
\hline Oligochaeta & - & 62 & 11 & - & 73 \\
\hline Chilopoda & - & 8 & 4 & - & 12 \\
\hline Hymenoptera & 2 & 172 & 3 & 80 & 257 \\
\hline Isoptera & 28 & 5 & - & - & 33 \\
\hline Total & 53 & 273 & 18 & 80 & 424 \\
\hline
\end{tabular}

O grupo Hymenoptera esteve presente nas quatro áreas estudadas com 2 indivíduos para área de savana natural, 172 indivíduos para área de frutíferas com 
práticas agroecológicas, 3 indivíduos na área de produção de hortaliças convencional e 80 indivíduos para a área degradada (Tabela 1). Estes resultados foram semelhantes aos registrados por Souza e colaboradores (2016) que observaram maior predominância do grupo Hymenoptera nos três diferentes ambientes estudos cultivados com coqueiro (Cocus nucifera.), feijoeiro macassar (Vigna unguiculata L.) e palma forrageira (Opuntia ficus-indica Mill.) no município de Parari, PB.

A densidade de Oligochaeta (Minhocas) foi diferenciada nas quatro áreas deste estudo. A área de fruticultura consorciada teve uma maior abundância desses indivíduos em relação às demais, sendo que área natural e área degradada não houve registro desse grupo taxonômico (Tabela 1). Esse fato pode estar relacionado com a grande quantidade de folhas depositadas no solo da área de fruticultura com manejo agroecológico, que possibilitou um microclima adequado à manutenção desse grupo de indivíduos, bem como a distribuição de indivíduos no solo. Marques et al. (2014) verificaram em pesquisa que a macrofauna edáfica apresenta-se mais abundante e rica na mata ciliar, sendo a ordem Oligochaeta o grupo taxonômico de maior frequência no solo desse ecossistema, evidenciando essa área como a de melhor equilíbrio ecológico em relação às monoculturas do café e do eucalipto.

A elevada frequência do grupo Hymenoptera na área de fruticultura com práticas agroecológicas (Tabela 1) pode ser explicada pelo manejo adotado, que promove manutenção dos resíduos culturais na superfície do solo, o que melhora o microclima, evita a exposição direta do solo aos raios solares e conserva a umidade. A intensidade de uso do solo, bem como o tipo de cobertura vegetal, constituiu fator determinante sobre a biodiversidade e abundância das comunidades de macroinvertebrados terrestres. Provavelmente o grande predomínio numérico relacionado à Ordem Hymenoptera em relação às outras ordens constatadas deve-se, segundo Agosti et al. (2000), ao fato das formigas apresentarem naturalmente uma grande dominância numérica em relação aos outros animais presentes em um habitat, totalizando mais de $10 \%$ da biomassa desses animais.

A maior densidade da macrofauna do solo foi observada na área de fruticultura com práticas agroecológicas com 281 indivíduos/m2 e a menor na área com produção de hortaliças convencional (18 indivíduos/m2) com uso de fertilizantes e pesticidas sintéticos (Tabela 2). A importância da macrofauna para a sustentabilidade dos agroecossistemas é inegável. Segundo Junior et al. (2010), a antropização de áreas 
utilizadas para a agricultura convencional interfere negativamente na dinâmica do ecossistema edáfico, em sua pesquisa os autores concluíram que as fertilizações químicas, aliadas ao manejo reduzido do solo, resultam em menores impactos sobre a diversidade de organismos participantes da ciclagem de nutrientes.A diversidade biológica foi avaliada pelo índice de Shannon (H) (Tabela 2), que trata do domínio dos grupos faunísticas nas áreas estudadas, em que os valores podem oscilar entre 0 e 5. A uniformidade foi avaliada pelo índice de Pielou (e), que representa a distribuição das ordens nas áreas estudadas. Constatou-se que a área degradada apresentou o menor índice de Shannon (1,06), apresentando apenas indivíduos da classe Hymenoptera. O uso da área como canteiro de obras ocasionou a compactação do solo o que levou ao empobrecimento do solo. De acordo com VARGAS et al. (2013), a maneira como o solo é usado, impõem restrições à alguns grupos de invertebrados e tendem a favorecer outros.

O Índice de Shannon indicou que a maior biodiversidade se encontra na área de fruticultura consorciada com práticas agroecológicas (Tabela 2). Os resultados do nosso estudo corroboram com SANTIAGO et al. (2013), que analisaram o comportamento dos índices de diversidade e uniformidade da macrofauna edáfica em sistemas irrigados de produção agroecológica e convencional de alimentos e concluíram que o manejo agroecológico do solo proporcionou um ambiente mais equilibrado em relação à área com manejo convencional. A implementação de práticas agroecológicas favoreceu o aumento na riqueza e densidade de espécies da macrofauna edáfica (Tabela 2), já que os maiores valores dessas variáveis foram observados nessa área.

Tabela 2 - Índice de Shannon e Pielou registrados em área de savana natural (SN), área de frutíferas consorciada com uso de práticas agroecológicas (AF), área de produção de hortaliças convencional (HC) e área degradada (AD) no município de Boa Vista-RR.

\begin{tabular}{|c|c|c|c|c|}
\hline Descrição & SN & $\mathbf{A F}$ & $\mathrm{HC}$ & AD \\
\hline Diversidade (Índice de Shannon) & 2,78 & 3,03 & 2,28 & 1,06 \\
\hline Índice de Shannon (antes <- elevado $\mathrm{e}^{\wedge}$ ) & 1,02 & 1,11 & 0,83 & 0,07 \\
\hline $\mathbf{N}^{\circ}$ grupos taxonômicos & 4 & 6 & 3 & 2 \\
\hline Índice Uniformidade Pielou & 0,74 & 0,62 & 0,75 & 0,10 \\
\hline $\mathrm{N}^{0}$ de indivíduos $\mathrm{m}^{2}$ & 53 & 281 & 18 & 81 \\
\hline
\end{tabular}


Conforme observado na Figura 1, houve diferença estatística (Tuckey 0,05) entre as áreas estudadas de savana natural $(\mathrm{SN})$, horta convencional $(\mathrm{HC})$, área degradada $(\mathrm{AD})$ e área de fruticultura $(\mathrm{AF})$ para a riqueza da macrofauna edáfica, indicando que o índice médio de indivíduos dentro das respectivas classes na área de fruticultura (AF) foi maior. Segundo Moço et al. (2005), a maior ou menor associação de um determinado grupo da fauna edáfica, em cada tratamento, deve-se ao tipo de preparo do solo e, principalmente, aos efeitos benéficos dos resíduos vegetais mantidos na superfície do solo, que proporcionam ambiente mais favorável à sobrevivência de determinados grupos. A diferença significativa entre as áreas pode estar relacionada à estrutura do local, como a cobertura do solo e as demais práticas utilizadas no manejo. Os ambientes heterogêneos oferecem maior quantidade de recursos (RIBAS et al., 2003; VARGAS et al., 2007), propiciando a existência de nichos diferentes e a diminuição de competição (RIBAS et al., 2007). Alves et al. (2008) sugere que a quantidade e qualidade da cobertura do solo e, ou, palhada também influencia a abundância e a diversidade da macrofauna do solo.

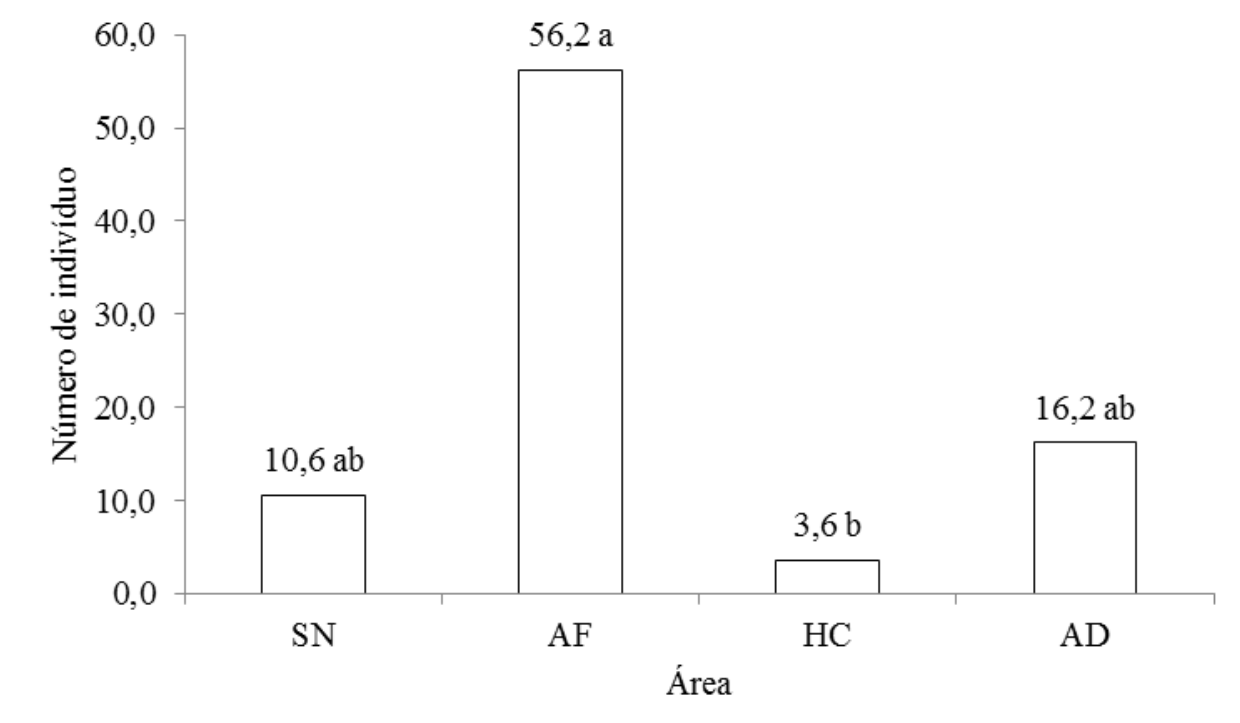

Figura 1- Distribuição do número médio de indivíduos de acordo com as áreas estudadas: área de savana natural (SN), área de frutíferas consorciada com uso de práticas agroecológicas (AF), área de produção de hortaliças convencional (HC) e área degradada (AD). Médias seguidas da mesma letra não diferem entre si pelo teste de Tukey a $5 \%$ de probabilidade. 
A área de horticultura convencional (HC) obteve a menor concentração de indivíduos evidenciando uma alteração na macrofauna do solo desta área conforme mostra a Figura 1. Santos et al. (2016) também constatou em sua pesquisa que o cultivo do solo com sistemas de produção de grãos, em plantio direto ou convencional, altera a estrutura da comunidade de macrofauna edáfica, em comparação à condição natural de Cerrado.

\section{CONCLUSÃO}

A macrofauna mostrou-se sensível ao manejo empregado nas áreas servindo como bioindicador da qualidade do solo.

A área de consórcio de frutíferas com uso de práticas agroecológicas apresentou maior diversidade, equitabilidade e riqueza em macrofauna.

\section{REFERÊNCIAS}

AGOSTI, D.; MAJER, J.D.; TENNANT, A.; SCHULTZ, T.R. (Eds.). Ants: Standard methods for measuring and monitoring biodiversity. Washington, D. C., USA: Smithsonian Institution Press,. 2000.

ALMEIDA, M. A. X.; SOUTO, J. S.; ANDRADE, A. P. Sazonalidade da macrofauna edáfica do Curimataú da Paraíba, Brasil. Revista Ambiência, v. 11, n. 2, p. 393-407, 2015 .

ALVARES, C. A.; STAPE, J. L.; SENTELHAS, P. C.; GONÇALVES, J. L. M.; SPAROVEK, G. Köppen's climate classification map for Brazil. Meteorologische Zeitschrift, v. 22, n. 6, p. 711-728. 2014.

ALVES , M. V.; SANTOS ,J. C. P.; GOIS, D. T.; ALBERTON „J. V.; BARETTA , D. Macrofauna do solo influenciada pelo uso de fertilizantes químicos e dejetos de suínos no Oeste do Estado de Santa Catarina. Revista Brasileira de Ciência do Solo. v. 32, p. 589-598, 2008.

ANDERSON, J. M.; INGRAM, J. S. I. Soil fauna. In: Tropical soil biological and fertility: A Handbook of methods. 2. ed. Wallingford: C.A. B. International, p. 44-46, 1993.

ARAÚJO, W. F.; ANDRADE JÚNIOR, A. S.; MEDEIROS, R. D.; SAMPAIO, R. A. Precipitação pluviométrica mensal provável em Boa Vista, Estado de Roraima, 
Brasil. Revista Brasileira de Engenharia Agrícola e Ambiental, v. 5, n. 3, p. 563-567, 2001.

BEGON, M.; HAPER, J. L.; TOWNSED, C. R. Ecology: individuals, populations and communities. Third ed. Oxford: Blackwell Science, 1996. 1068 p.

BRADY, N.C.; WEIL, R.R. Elementos da natureza e propriedades dos solos. Porto Alegre: Bookmann Editora LTDA, 2013.

DUARTE, O. R.; ALBUQUERQUE, T. C. S. de.; MATTHIENSEN, A.; CHAGAS, E. A.; KAMINSKI, P. E. Compatibilização de Demandas para a Agricultura Familiar no Estado de Roraima. Boa Vista: Embrapa Roraima, 2009. 42p. (Embrapa Roraima. Documentos, 30).

FERREIRA, D. F. Sisvar: a Guide for its Bootstrap procedures in multiple comparisons. Ciência e Agrotecnologia, v. 38, n. 2, p. 109-112, 2014.

KORASAKI, V.; MORAIS, J. W. de; BRAGA, R. F. Macrofauna. In: MOREIRA. F. M. S.; CARES, J. E.; ZANETTI, R.; STÜRMER, S. L. (Eds.). O ecossistema solo: componentes, relações ecológicas e efeitos na produção vegetal. Lavras: Editora da UFLA, p. 79-128.2013.

LAVELLE, P.; DECAËNS, T.; AUBERT, M.; BAROT, S.; BLOUIN, M.; BUREAU, F.; MARGERIE, P.; MORA, P. E ROSSI, J.P. Soil invertebrates and ecosystem services. European Journal of Soil Biology, v. 42, Supplement 1, p. S3-S15, 2006.

MARQUES, D. M.; SILVA; A. B.; SILVA, L. M. MOREIRA, E.A.; PINTO , G. S. Macrofauna edáfica em diferentes coberturas vegetais. Bioscience Journal, v. 30, p. 1588-1597, 2014.

MOÇO, M. K. et al. Caracterização da fauna edáfica em diferentes coberturas vegetais na região norte fluminense. Revista Brasileira de Ciência do Solo, Viçosa, v. 29, n. 4, p. 555-564, 2005.

JÚNIOR, L.R.P.; FERRAZ, D. DE S., ALVES, G.S., SOUSA, J. da S., SOUTO, J.S. Influência do cultivo agrícola convencional nas características químicas $e$ macrofauna edáfica. Engenharia Ambiental, v.7, n.3, p.166-177, 2010.

QUERINO, R. B.;JÚNIOR, A. L. M.; VIEIRA, B.de A. H.; SANTOS,C. S. V. dos. LUZ, F. J. F.;ZILLI, J. E.; NECHET,K. de L.; COSTA,M. C. G.; MATTOS,P. S.R. de.; MEDEIROS,R. D.de. Diagnóstico de pequenas propriedades hortifrutigranjeiros em Boa Vista/RR ; Boa Vista, RR: Embrapa Roraima, 2008. 23 p.

RIBAS, C.R.; SCHOEREDER, J.H.; PIC, M.; SOARES, S.M. Tree heterogeneity, resource availability, and large scale processes regulating arboreal ant species richness. Austral Ecology, v. 28, n. 3, 2003.

SANTIAGO ,F. S.; NANES , M. B., JALFIM ; F. T., DIAS ,I. C. G. M.; BLACKBURN ,R. M.; SILVA , N.C. .G; AZEVEDO, M. A. A.; FREITAS , R. R. L.. - 
Influência do manejo agroecológico na macrofauna edáfica em área de hortaliças e frutas no Sertão do Apodi-RN. IN:X Congresso Nacional de Meio Ambiente de Poços de Caldas - Poços de Caldas/MG, 2013.

SANTOS, D. P. ; SANTOS, G. G. ; SANTOS, I. L. ; SCHOSSLER, T. R. ; NIVA, C. C. ; Marchão, R.L . Caracterização da macrofauna edáfica em sistemas de produção de grãos no Sudoeste do Piauí. Pesquisa Agropecuária Brasileira, v. 51, p. 1466-1475, 2016.

SILVA, R. F.; TOMAZI, M.; PEZARICO, C. R.; AQUINO, A. M.; MERCANTE, F. M. Macrofauna invertebrada edáfica em cultivo de mandioca sob sistemas de cobertura do solo. Pesquisa Agropecuária Brasileira, v. 42, n. 6, p. 865-871, 2007.

SILVA, A. R. de A.. A cadeia produtora de hortifrúti em Boa Vista, Roraima: produtores, consumidores e qualidade pós - colheita dos principais produtos. I Antonia Raniely de Almeida Silva. Boa Vista - RR: UERR, 2016. 91f. il. $30 \mathrm{~cm}$. Dissertação (Mestrado) - Universidade Estadual de Roraima.

SOUZA, J. T. A.; FARIAS, A. A.; FERREIRA, R. C. C.; OLIVEIRA, S. J. C.; CAVALCANTE, L. F.; FIGUEIREDO, L. F.; CORREIA, F. G. Macrofauna edáfica em três ambientes diferentes na região do Cariri Paraibano, Brasil. Revista Scientia Agraria Paranaensis, v. 15, n. 1, p. 94-99, 2016.

SOUZA, J. T. A.; OLIVEIRA, S. J. C.; NÁPOLES, F. A. M.; SOUZA, M. S.; MEDEIROS, M. R. Diversidade de macrofauna edáfica em diferentes ambientes de cultivo no agreste da Paraíba, Brasil. Revista de Agricultura Neotropical, v. 4, n. 3, p. 55-60, 2017.

STORER, T.I.; USINGER, R. L.; STEBBINS, R. C.; NIBAKKEN, J.W. Zoologia Geral. $6^{\text {a }}$ ed. São Paulo: Editora Nacional. 2000.

VARGAS, A.B.; CHAVES, D.A.; VAL, G.A.; SOUZA, C.G.; FARIAS, R.M.; CARDOZO, C.; MENEZES, C.E.G. Diversidade de artrópodes da macrofauna edáfica em diferentes usos da terra em pinheiral, Acta Scientiae \& Technicae, v.1, 2013.

VARGAS, A.B.; MAYHÉ-NUNES, A.J.; QUEIROZ, J.M.; SOUZA, G.O.; RAMOS, E.F. Efeitos de Fatores Ambientais sobre a Mirmecofauna em Comunidades de Restinga no Rio de Janeiro, RJ. Neotropical Entomology. v. 36, n. 1, 2007. 